\title{
Model Based Vehicle Localization via an Iterative Parameter Estimation
}

\author{
Máté Fazekas, Balázs Németh $\left.{ }^{(}\right)$, and Péter Gáspár \\ Systems and Control Laboratory, Institute for Computer Science and Control, \\ Hungarian Academy of Sciences, Kende u. 13-17, Budapest 1111, Hungary \\ \{mate.fazekas, balazs.nemeth, peter.gaspar\}@sztaki.mta.hu
}

\begin{abstract}
This paper proposes a novel method for the estimation of the wheel circumferences, which have significant effects on a vehicle model based localization. One of the advantages of the method is that only cost effective onboard sensors, such as GPS, magnetometer, IMU and wheel encoders are used. Moreover, the estimation methods based on pure vehicle models can result in suitable localization, when other solutions are not effective i.e. the GPS signals are not available or other sensors are inaccurate, such as IMU measurements with low, constant velocity. The presented off-line algorithm has three main layers connecting the Kalman-filter and Least Squares based estimation processes in an iterative way. During the procedure the side-slip is estimated, which has a significant impact on the dynamics of the vehicle and the further estimations. Since in the method all of the measurements are used at once and the side-slip is also calculated, a highly accurate identification with low sensitivity on the noise can be reached. The efficiency of the vehicle model calibration is presented through CarSim simulations.
\end{abstract}

Keywords: Autonomous localization - Estimation - Kalman filter · Self-calibration

\section{Introduction and Motivation Example}

Vehicle localization became a widely research topic in the automotive industry with appearing of the autonomous vehicle functions. Several methods for localization were presented in the recent years using a wide range of sensors, such as camera, LiDAR, GPS, IMU and wheel encoders. The perception based methods require prior teaching or well recognizable features, see [1]. The fusion of GPS and IMU measurements could be precise on higher velocity scenarios, but is assumes the actual knowledge of the covariances of the signals. Moreover, the signals of the GPS are not available in parking garages or in several urban areas, e.g. next to the buildings with high walls. Therefore, in these situations the wheel encoder based odometry can be an appropriate choice for vehicle localization, see [2]. However, these localization methods require the model of the vehicle, 
which can contains parameter uncertainties. Thus, the estimation of some vehicle parameters is an important feature of the autonomous vehicle localization, which is a challenge in the research.

The odometry based on the vehicle model has widely used in mobile robot applications in the last few years i.e. in parking assist functions [3] and in the automated navigation part of a fusion algorithm [4]. In the odometry based navigation systems the well-calibrated vehicle model is required to ensure the highly accurate pose estimation. Proposed calibration methods can be found in [5] and [6], but the full automated self-calibration is a novel research area. The absolute pose measurements are suitable to estimate the real value of the parameters. A self-adaptive method is presented in [7], which deals with the estimation of the time delay of the sensor.

The parameter identification problem is handled with the least squares approximation, while the optimal state estimation at various environmental conditions is guaranteed by Kalman-filters explained in detail in [8]. In the nonlinear case a possible solution can be the Extended Kalman-filter, see in [6] and [7].

The scope of this paper is to propose a novel iterative parameter estimation method for optimal calibration of wheel encoder based vehicle models. Parameter identification of wheel circumferences are performed based on an algorithm. Since the variation of the wheel circumference has a low dynamics, the off-line estimation can be enough. The advantages of the method is that it is not necessary to find a balance between the computation time and the preciseness of the estimation due to the off-line computation. In the method an iterative procedure is proposed to improve the accuracy of the vehicle model setting. The proposed method can have several other application areas in the autonomous vehicle control, determination of the IMU signal bias and covariance values or it can provide a prior information for the perception methods.

In the following examples the impact of the wheel radius on the vehicle motion is illustrated in Fig. 1, through a parking-garage maneuvering scenario with various tyre wear values. Figure 2 contain the used tyre wear $(R L$ and $R R$ means rear-left and rear right) and the results, which show that the small

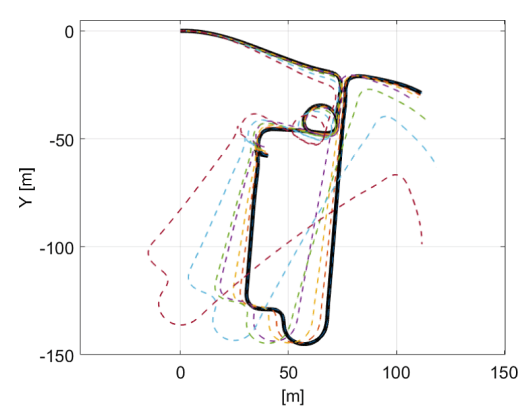

Fig. 1. Odometry paths

\begin{tabular}{|c|c|c|c|c|c|}
\hline \multicolumn{2}{|c|}{ Tyre wear } & \multicolumn{4}{|c|}{ Errors } \\
\cline { 3 - 6 } & \multicolumn{2}{|c|}{ Position } & \multicolumn{2}{|c|}{ Orientation } \\
\hline $\begin{array}{c}\text { RL } \\
{[\mathrm{mm}]}\end{array}$ & $\begin{array}{c}\text { RR } \\
{[\mathrm{mm}]}\end{array}$ & $\begin{array}{c}\text { Mean } \\
{[\mathrm{m}]}\end{array}$ & $\begin{array}{c}\text { Max } \\
{[\mathrm{m}]}\end{array}$ & $\begin{array}{c}\text { Mean } \\
{[\mathrm{deg}]}\end{array}$ & $\begin{array}{c}\text { Max } \\
{[\mathrm{deg}]}\end{array}$ \\
\hline 0 & 0 & 0.34 & 0.77 & 0.53 & 4.08 \\
\hline 1.5 & 1.5 & 1.26 & 3.66 & 1.54 & 4.95 \\
\hline 3 & 3 & 2.61 & 7.50 & 2.89 & 7.44 \\
\hline 6 & 6 & 5.37 & 15.24 & 5.79 & 12.55 \\
\hline 3.1 & 2.9 & 6.33 & 19.01 & 8.33 & 16.39 \\
\hline 3.25 & 2.75 & 12.90 & 36.39 & 16.47 & 33.44 \\
\hline 3.5 & 2.5 & 24.19 & 70.04 & 30.16 & 62.34 \\
\hline
\end{tabular}

Fig. 2. Tyre wear and errors 
difference from the true wheel radius can lead to a significantly different course of the autonomous vehicle. Thus the wheel circumference is a crucial parameter in the model of the on-line odometry, therefore the off-line calibration has high priority.

The paper is organized as follows. In Sect. 2 the two-wheel model of the odometry is presented. Moreover, the iterative parameter identification method is found in Sect.3, while the tuning of the algorithm is proposed in Sect. 4 . Simulation results are presented in Sect. 5 and finally, the contributions of the paper and the future challenges are summarized in Sect. 6.

\section{Model Based Vehicle Localization}

The dead reckoning navigation is based on a model, of which estimated state vector $\mathbf{x}_{k}$ contains the longitudinal and lateral vehicle positions $x_{k}, y_{k}$ and the heading angle $\theta_{k}$. The accuracy of the model highly depends on the calibration of the kinematic odometry parameters. The planar motion of the vehicle is calculated from the vehicle velocity $v_{k}$ and the angular rate $\omega_{k}$, such as

$$
\begin{aligned}
& x_{k}=x_{k-1}+v_{k} \cdot \Delta t \cdot \cos \left(\theta_{k-1}+\omega_{k} / 2 \cdot \Delta t+\beta_{k}\right) \\
& y_{k}=y_{k-1}+v_{k} \cdot \Delta t \cdot \sin \left(\theta_{k-1}+\omega_{k} / 2 \cdot \Delta t+\beta_{k}\right) \\
& \theta_{k}=\theta_{k-1}+\omega_{k} \cdot \Delta t
\end{aligned}
$$

where $\beta_{k}$ is the side-slip angle and $\Delta t$ is sampling time. Moreover, $v_{k}$ and $\omega_{k}$ are calculated using the two-wheel vehicle model $(2 \mathrm{~W})$, where the velocities are determined by the rear wheel speeds, which are resulted by the product of wheel rotation measurements $n_{i, k}$ and the circumference $c_{i}, d_{i, k}=n_{i, k} \cdot c_{i}$ such as

$$
v_{k}=\left(d_{R L, k}+d_{R R, k}\right) /(2 \cdot \Delta t) \quad \omega_{k}=\left(d_{R R, k}-d_{R L, k}\right) /\left(t_{R} \cdot \Delta t\right)
$$

Thus, the accuracy of the model-based localization highly depends on the $c_{i}$.

\section{Kalman-Filter Based Iterative Least Square Parameter Estimation Method}

The iterative estimation method is based on the presented vehicle model, and the measurement of GPS position, magnetic heading, IMU acceleration and yaw rate and wheel rotation velocity. The process of the iterative solution is illustrated in Fig. 3. The method has three main layers, in which the Kalman-filtering (KF) and the Least Squares (LS) optimization are connected together in an iterative way. This approach can also be feasible for identification of Hammerstein and Wiener models, see in [11]. 


\subsection{Reference Calculation and Side-Slip Estimation}

In the first layer reference pose values $\mathbf{r}_{k}=\left[\tilde{x}_{k}, \tilde{y}_{k}, \tilde{\theta}_{k}\right]^{T}$ are calculated using the GPS and IMU measurements, which are considered as references for the computation. This fusion method has been investigated already in a wide range of papers considering the dynamic equation of $\ddot{p}=a$, where $p$ is the position and $a$ is the acceleration. The implemented method is similar to [9]. The side-slip is assumed to be high impact on the precise estimation of the states. Thus, the measured signals are applied in a KF based side-slip estimation presented in [10].

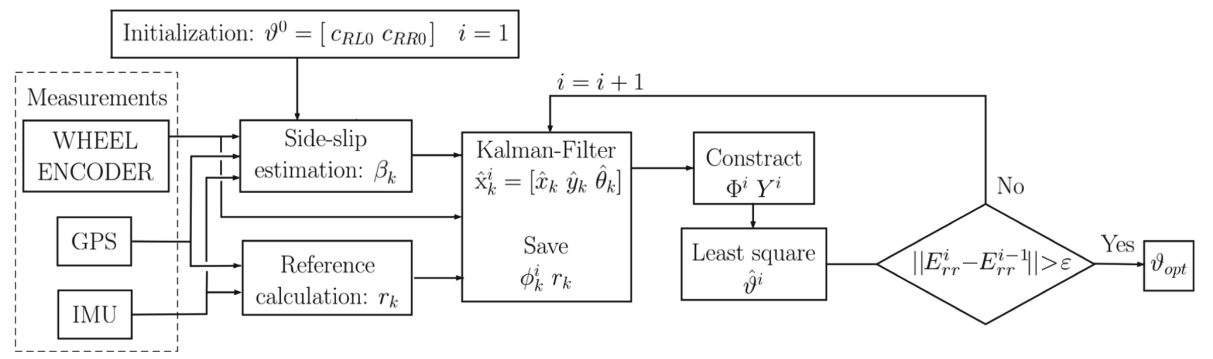

Fig. 3. Process of the iterative parameter estimation

\subsection{State Estimation with Kalman-Filter and Least Square Based Parameter Identification}

The core of the method is an iterative loop of state estimation of the vehicle and parameter identification of the wheel circumferences.

First, the state vector containing the vehicle pose as $\mathbf{x}_{k}=\left[x_{k}, y_{k}, \theta_{k}\right]^{T}$ is filtered with an Extended Kalman-filter (EKF), which uses the two-wheel vehicle model as state function, the wheel encoders as inputs $\left(d_{i, k, n}=n_{i, k} \cdot c_{i, n}\right)$ and the reference position and orientation values as measurements such as

$$
\mathbf{x}_{k}=\boldsymbol{f}\left(\mathbf{x}_{k-1}, \mathbf{u}_{k-1}\right) \quad \mathbf{u}_{k-1}=\left[\begin{array}{ll}
d_{R L, k-1, n-1} & d_{R R, k-1, n-1}
\end{array}\right]^{T} \quad \mathbf{y}_{k}=\mathbf{r}_{k}
$$

where the wheel circumferences are the estimated values from the previous step. The filtering of $\widehat{\mathbf{x}}_{k}=\left[\widehat{x}_{k}, \widehat{y}_{k}, \widehat{\theta}_{k}\right]^{T}$ is performed in the following two phase.

- Prediction based on the model The predicted states and covariance matrix are calculated as follows

$$
\widehat{\mathbf{x}}_{k}^{-}=\boldsymbol{f}\left(\widehat{\mathbf{x}}_{k-1}, \mathbf{u}_{k-1}\right) \quad \Sigma_{k}^{-}=F_{k} \Sigma_{k-1} F_{k}^{T}+P
$$

The method handles the nonlinearity with the calculation of the approximate derivatives as the first order Taylor linearization, thus the Jacobian is stated as follows and compute in the previous state as

$$
F_{k}=\left.\frac{\partial \boldsymbol{f}(\mathbf{x}, \mathbf{u})}{\partial \mathbf{x}}\right|_{\mathbf{x}=\widehat{\mathbf{x}}_{k-1}, \mathbf{u}=\mathbf{u}_{k-1}}
$$


- Innovation based on the measurements In the innovation phase the measurements are used to improve the estimation. The state and the covariance calculated as follows

$$
\widehat{\mathbf{x}}_{k}=\widehat{\mathbf{x}}_{k}^{-}+G_{k}\left(\mathbf{y}_{k}-\boldsymbol{h}\left(\widehat{\mathbf{x}}_{k}^{-}\right)\right) \quad \Sigma_{k}=\left(I-G_{k} H_{k}\right) \Sigma_{k}^{-}
$$

where $\boldsymbol{h}(\mathbf{x})$ is the measurement equation, which is $\boldsymbol{h}(\mathbf{x})=\left[x_{k}, y_{k}, \theta_{k}\right]^{T}$. The Jacobian is stated as $H_{k}=I . G_{k}$ is the Kalman-Gain factor, which ensures the optimal estimation of the states and guarantees the minimum covariance. The gain equation follows as

$$
G_{k}=\Sigma_{k}^{-} H_{k}^{T}\left(H_{k} \Sigma_{k}^{-} H_{k}^{T}+M\right)^{-1}
$$

The filter design requires the $P$ and $M$ matrixes, which are the model and measurements covariances. These determine the ratio of input and measurement in the optimal fused state, therefore need to be adjusted properly. The detailed tuning method can be found in the next section.

The optimization of the parameters $c_{R L}$ and $c_{R R}$ is based on the least square regression method, because linear algebraic equations can be formed between the results of the previous estimations and the identified values, such as

$$
\begin{gathered}
\phi_{k}=\left[\begin{array}{cc}
\frac{n_{R L, k}}{2} \cdot \cos \left(\widehat{\theta}_{k-1}\right) & \frac{n_{R R, k}}{2} \cdot \cos \left(\widehat{\theta}_{k-1}\right) \\
\frac{n_{R L, k}}{2} \cdot \sin \left(\widehat{\theta}_{k-1}\right) & \frac{n_{R R, k}}{2} \cdot \sin \left(\widehat{\theta}_{k-1}\right) \\
-\frac{n_{R L, k}}{t_{R}} & \frac{n_{R R, k}}{t_{R}}
\end{array}\right] \\
\underbrace{\left[\mathbf{r}_{k}-\widehat{\mathbf{x}}_{k-1}\right]}_{Y}=\underbrace{\phi_{k}}_{\Phi} \boldsymbol{\vartheta} \widehat{\boldsymbol{\vartheta}}_{\text {opt }}=\left(\Phi^{T} W \Phi\right)^{-1} \Phi^{T} W Y
\end{gathered}
$$

where $\boldsymbol{\vartheta}=\left[c_{R L}, c_{R R}\right]^{T}$ contains the wheel circumferences, $Y$ consist the reference measurements and the estimated states and $\Phi$ is based on the two-wheel model. In the equation of the resulted solution of the optimal parameter, $W$ is a positive weighting matrix with the coefficients of $\operatorname{diag}\left(\left[\begin{array}{lll}1 & 1 & 10\end{array}\right]\right)$ because of the compensation of $1 \mathrm{~m}$ position and $1 \mathrm{rad}$ orientation errors.

\section{Tuning of the Algorithm}

The two main goal of the tuning procedure are the ensuring of the convergence to the global optimum setting and the guarantee of robustness of the method to perform the calibration in an automatic way.

The $P$ and $M$ covariance matrices of the EKF symbolize the variance of the model states predicted from the inputs and the variance of the measurements. Generally the values are set to the noise of the used sensors. In the most application of the filter the covariances are fix and contain prior calculated values. This results deviation from the optimal, if the actual noise is different from the precalculated. Nevertheless the determination of actual sensor noises online is a highly difficult problem. The value of the $G$ Kalman-gain mostly depends on the 
ratio of the matrices not on the exact numeric values. However in our methods the EKF operates in an iterative loop, where the input is changing, because of the continuous modification of the wheel circumferences see in the (3). Thus varying covariance is assumed to be required. Taking into consideration that our main goal is the optimal calibration of the vehicle model, the $2 \mathrm{~W}$ model with the estimated parameters is integrated on the whole track without any fusion in every steps. The deviation from the calculated reference positions is determined and the parameter accuracy is evaluated using this error.

Furthermore the changing of error values is used for tuning. Ten different noise cases are generated and added to the simulated driving scenario mentioned in the Sect. 5. From the noise-free case optimal values of the vehicle model setting, parameters and covariance matrices can be calculated. However in real cases the determination is impossible. To deal with this problem varying covariance setting is proposed. A pre-calculation is assumed and used as initial value. The convergence towards the optimal setting is guaranteed through the appropriate decreasing of $\mathrm{P}$ and $\mathrm{M}$ matrices with the following schema

$$
\begin{gathered}
M=\operatorname{diag}\left(\left[\begin{array}{lll}
\frac{10}{\left(q_{M}\right)^{i}} & \frac{10}{\left(q_{M}\right)^{i}} & \frac{0.1}{\left(q_{M}\right)^{i}}
\end{array}\right]\right) \\
P=\operatorname{diag}\left(\left[\begin{array}{lll}
10-q_{P} \cdot i & 10-q_{P} \cdot i & 0.1-q_{P} \cdot i
\end{array}\right]\right)
\end{gathered}
$$

where $i$ is the actual iteration number and $q$ values are variables in the range of $1 \leq q_{M} \leq 1.5$ for $q_{M}$ and $0.2 \leq q_{P} \leq 0.7$ for $q_{P}$, taking into account that the covariance values must be positive. The reason of the selection is that in first steps the model probably inaccurate due to the uncertain parameter values, therefore relative higher decreasing for the $M$ is suggested.

The efficiency of the proposed selection is presented in Fig. 4, where the same noise cases are used, but the covariances are 3 times higher and lower than the optimal. The mentioned position error values of the integrated $2 \mathrm{~W}$ model with the estimated parameters without fusion from the calculated reference pose are determined. The varying method is compared with a fix setting with values of the initial of the varying. Using the optimal covariance setting the varying leads also to better results, the mean deviation is $68.6 \%$ higher than the error of optimal model setting for the varying and $80.9 \%$ higher for the fix selection. Using the non-optimal covariance values the mean deviations are increasing with $8.7 \%$ and $144.4 \%$ in the method of fix and with $17.2 \%$ and $6.2 \%$ in the method of varying for the $3 P_{o p t}$ and $1 / 3 P_{\text {opt }}$ case. The modification of the measurement covariance is similar with the illustrated cases as $1 / 3 M_{\text {opt }} \sim 3 P_{\text {opt }}$ and $3 M_{\text {opt }} \sim 1 / 3 P_{\text {opt }}$. Therefore the presented varying method can compensate the non-optimal setting of the covariances.

In the proposed iterative method parameter identification is performed in every iteration steps and the resulted values are assumed to be the optimal because of the least square method. However in the presented algorithm the LS problem is changing in every iteration step, because in the (9) the $Y$ and $\Phi$ depend on the wheel circumferences. This means that in an iteration step the LS method estimate the optimal parameters of the actual LS problem, but with 


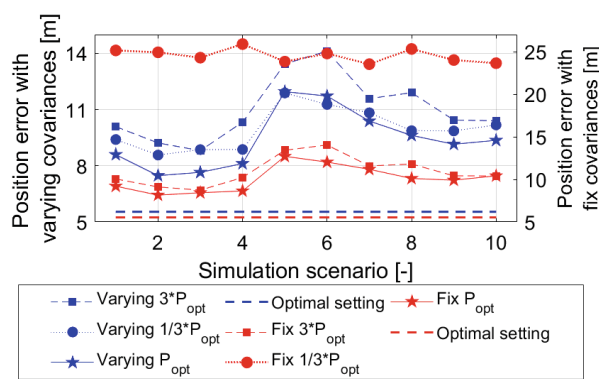

Fig. 4. Effect of covariances

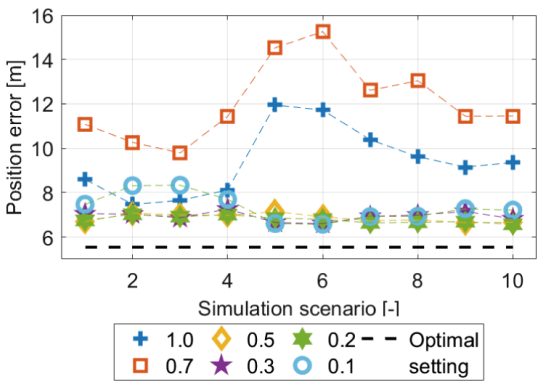

Fig. 5. Effect of $\alpha$

the estimated values an other LS problem is generated, where an other optimal parameter vector is existed. The deviation between the problems is a crucial question. To ensure the convergence to the global optimum vehicle setting the new parameter values are only a ration of the estimated actual optimal such as,

$$
\widehat{\boldsymbol{\vartheta}}_{n}=\widehat{\boldsymbol{\vartheta}}_{n-1}+\alpha\left(\widehat{\boldsymbol{\vartheta}}_{o p t, n}-\widehat{\boldsymbol{\vartheta}}_{n-1}\right)
$$

where $\alpha$ is in the range of $0<\alpha \leq 1$. The idea is based on the learning rate application in the gradient based optimization methods. The effect of $\alpha$ is illustrated in the Fig. 5, with using of the previous mentioned varying covariance process. It can be seen that with lower values than 0.5 the position error is extremely decreasing, however with too small value as 0.1 the error is increasing again. Furthermore, with the application of the ratio the deviation from the optimal vehicle setting is decreased to $22.5 \%$ with the optimal covariances and with the modificated matrices the increase of the errors are only $2.9 \%$ and $4.7 \%$ using the varying process. Thus these two developed tuning processes ensure the convergence to the optimal vehicle setting and handle the problem of non-optimal covariance application, therefore the identification process could be an automatic method.

\section{Simulation Results}

The efficiency of the proposed estimation method is demonstrated through various simulation examples, using the high-fidelity vehicle dynamic software CarSim. It is assumed that the measured signals contain noises, which have Gaussian distribution. The E-class test vehicle was driven on the Michigan Waterford Course with $60 \mathrm{~km} / \mathrm{h}$. The initial values of the parameters are $2.2 \mathrm{~m}$ in every case. The track and the path of the $2 \mathrm{~W}$ odometry model with the initial nominal parameters can be found in Fig. 7. The length of the route is $2.2 \mathrm{~km}$ and contains about a dozen bends. As we can see the calculated path is not fit to the reference, the position error is more than $30 \mathrm{~m}$. The advantages of the simulation is that the optimal vehicle model setting is known, this is appeared on the figures with the legend label of 'Optimal model', but this value is unknown in real 
situations. However the calculated position errors are determined using the calculated reference by the Kalman-filters mentioned in Sect. 3.1, and based on the measured signals only. Thus these errors can be used to manage the estimation algorithm.

The identification process is performed in 10 scenarios with 9 iteration steps and the Scenario 5 is presented in the Fig. 6. The lower graph shows that the deviation of the calculated $2 \mathrm{~W}$ model path with the actual estimated parameters from the calculated reference is decreasing continuously in the first 4 iteration. The deviation of the position error with the resulted optimal wheel circumferences is about $2 \mathrm{~m}$ from the error of the optimal vehicle model setting. After the 4th step the pose errors are increasing, however the estimation can be stopped due to the knowledge of the errors. The path using the estimated vehicle model is illustrated in the Fig. 7. The fit is almost perfect considering that a vehicle model, which uses only the two rear wheel encoders, is integrated over $2 \mathrm{~km}$.

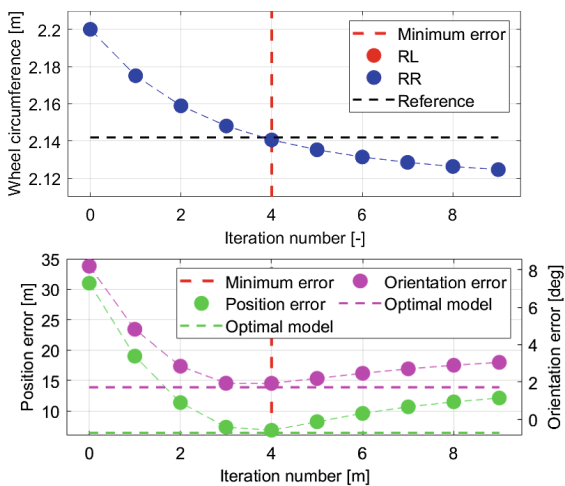

Fig. 6. Results of iteration in Scenario 5 .

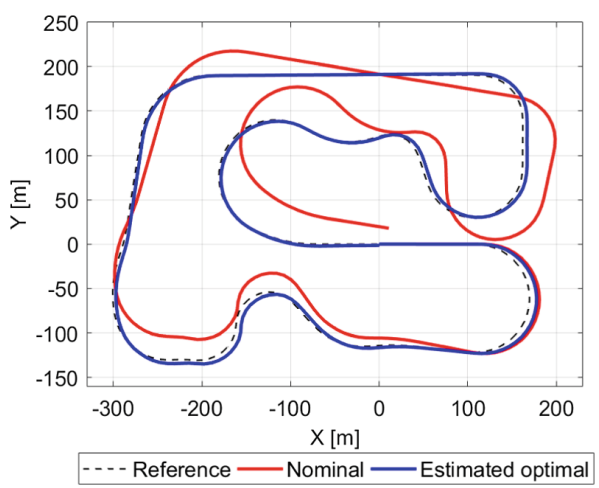

Fig. 7. Track of estimation in Scenario 5 .

The characteristics of the estimation is similar to the other cases, only the place of the optimum can be differed by a few steps. The average error of the scenarios is $6.7 \mathrm{~m}$, which is higher only with $1.2 \mathrm{~m}$ than the optimal setting. The robustness of the tuning method is symbolized with the low mean absolute deviation from the average with the $13 \mathrm{~cm}$ value. Figure 8 presents the results of the wheel circumference estimation using the proposed iterative off-line method. Since the off-line iteration uses all of the measured signals at once, it has a small sensitivity on the noise. The mean estimation errors from the reference, which results the optimal vehicle setting are $3.41 \mathrm{~mm}$ and $3.46 \mathrm{~mm}$ for the $c_{R L}$ and $c_{R R}$, thus the deviation can be reduced under $0.2 \%$, which results in an outstanding performance.

Up to this point the side-slip is assumed to be zero $\beta_{k} \approx 0$ in the estimation procedure. Taking into consideration this dynamic variable the result of the mentioned method in Sect. 3.1 is included in the algorithm at the model equations. 


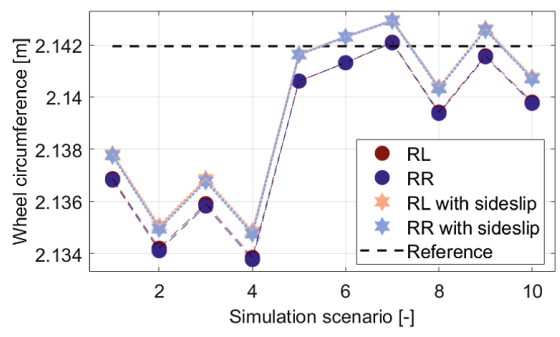

Fig. 8. Wheel circumferences of scenarios

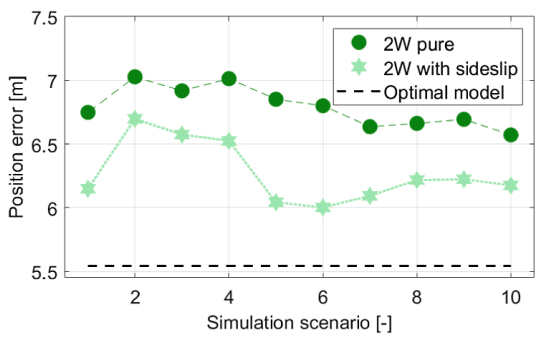

Fig. 9. Position errors of scenarios

The Figs. 8 and 9 also show the identified parameters and the errors. The parameter deviation decreased with $0.8 \mathrm{~mm}$, which results $0.5 \mathrm{~m}$ closer to the optimal setting, thus the deviation from it is only $13 \%$, therefore it is evaluated that well enough vehicle model calibration is attended for autonomous localization from only a $2.2 \mathrm{~km}$ long measurement.

\section{Conclusions}

The paper proposed a parameter identification algorithm for wheel circumferences of two-wheel vehicle model. The method has three layers. First, reference signals are calculated, which are fusioned with the $2 \mathrm{~W}$ odometry model in a Kalman-filter in the second layer. Thirdly, the core of the process is a least squares based parameter estimation. The process runs in an iterative loop, where the convergence to the global optimum vehicle setting is guaranteed by a tuning method with various covariance matrices and a ratio parameter in the direction of estimation. Since the off-line methods uses all of the measurements at once, a highly accurate estimation with low sensitivity on the noise can be reached. The efficiency of the algorithm is presented through CarSim simulations.

As a future challenge, the applied vehicle odometry model might be improved through the consideration of tyre radius varying in corners and the variation of the vertical load can be considered.

Acknowledgement. This work has been supported by the grant '2018-1.2.1-NKP00008: Exploring the Mathematical Foundations of Artificial Intelligence'. This paper has been partially supported by the János Bolyai Research Scholarship of the Hungarian Academy of Sciences and the ÚNKP-18-4 New National Excellence Program of the Ministry of Human Capacities.

\section{References}

1. Bloesch, M., et al.: Robust visual inertial odometry using a direct EKF based approach. In: IEEE/RSJ International Conference on Intelligent Robots and Systems, Hamburg, Germany, September 2015 
2. Funk, N., Alatur, N., Deuber, R., et al.: Autonomous electric race car design. In: EVS30 Symposium, Stuttgart, Germany, October 2017

3. Brunker, A., et al.: Odometry 2.0: a slip-adaptive UIF-based four-wheel-odometry model for parking. IEEE Trans. Intell. Transp. Syst. 4(1), 114-126 (2019)

4. Thrun, S., et al.: Stanley: the robot that won the DARPA grand challenge. J. Field Robot. 23(9), 661-692 (2006)

5. Kooktae, et al.: Calibration of kinematic parameters of a car-like mobile robot to improve odometry accuracy. In: IEEE International Conference on Robotics and Automation, Pasadena, California, May 2008

6. Larsen, T., et al.: Location estimation for an autonomously guided vehicle using an augmented Kalman filter to autocalibrate the odometry, August 1998

7. Brunker, A., et al.: GNSS-shortages-resistant and self-adaptive rear axle kinematic parameter estimator. In: IEEE Intelligent Vehicles Symposium, LA, USA, June 2017

8. Crassidis, J.L., et al.: Optimal Estimation of Dynamic Systems. CRC Press, Boca Raton (2017)

9. Caron, F., et al.: GPS/IMU data fusion using multisensor Kalman-filtering: introduction of contextual aspects. Inf. Fusion 7, 221-230 (2006)

10. Bevly, D.M., et al.: Integrating INS sensors with GPS measurements for continuous estimation of vehicle sideslip, roll, and tire cornering stiffness. IEEE Trans. Intell. Transp. Syst. 7(4), 483-493 (2006)

11. Ma, J., et al.: Combined state and parameter estimation for Hammerstein systems with time delay using the Kalman filtering. Int. J. Adapt. Control Signal Process. 31, 1139-1151 (2017) 